\title{
22 Conclusions: What Have We Learned?
}

This book has presented both qualitative and quantitative analyses of the writings left by suicides in their diaries, suicide notes, poems, letters, twitter feeds, blogs and tape recordings. My first reaction was how powerful the diaries that I have read are, and this is especially true for the diaries that I was given by those bereaved by the suicide of a loved one. The writers of those diaries present in clear, emotional and moving words the conflicts that they are experiencing. Compared to these diaries, suicide notes are very brief and often fail to provide any clues to what was in the minds of those about to kill themselves. The diaries provide scores, even hundreds, of pages describing the writer's pain.

Let me review my thoughts on this project.

\subsection{Biographies versus Diaries}

I started this project in order to satisfy myself. If my research, and the research of others, fails to provide an insight into the mind of the suicidal individual, might the study of their diaries provide this insight? I had read biographies of famous individuals who had taken their own lives, and these provide some clues about the life paths that end in some people killing themselves. But biographies are written by an outsider, someone who knows the facts about, but not the mind of, the protagonist. The diaries give us a window into their mind.

The facts of a person's life are, of course, important, but combining the facts with the words of the protagonist is immeasurably better. For example, the biography of Cesare Pavese by Lajolo (1983) (discussed in Chapter 4) is important in framing the life of Pavese. Pavese's novels, which reflect his personal life, also provide insight into his mind. But his diary, despite the translation and possible editing, gives us insight into his thought processes which illuminate the facts of his life, information which is not present in Lajolo's biography of Pavese or in Pavese's own novels.

Of course, for the personal diaries given to me by the significant others of those who died by suicide, there is no biography. In my analysis of the letters written by Clara (Chapter 16), a biography of her life would have provided better insights into the psychodynamics motivating her choice of suicide. But no detailed biographies are available for the writers of these private diaries, only the sketchy outline of their life paths.

The published diaries (such as that from Cesare Pavese) are typically accompanied by extensive biographies, but these diaries may have been written by those who knew that their diaries would eventually be published. Thus, they may have been edited by the writer, in addition to edits made by the scholar who presented them for publication. Thus, they are likely to be more constrained and more intellectual in tone. 


\subsection{Qualitative versus Quantitative Methodologies}

The chapters in this book have used both clinical insight and quantitative analysis. My clinical analysis of the diary of the university professor (Robert in Chapter 7) provides a detailed description of the conflicts and psychological pain that Robert was experiencing, and it is surprising to me that he lived as long as he did. For some of the diaries, it was possible also to run the text through the LIWC program. Sometimes, this confirmed the clinical insights, as when it clearly identified the increase in positive emotions and the decrease in negative emotions over time in Katie's diary (Chapter 3), which was noted impressionistically by Silvia Canetto (2004) in her chapter in my book Katie's Diary (Lester, 2004b). On occasions, the LIWC identified trends that a simple reading of the diaries would not identify. For example, the increase in question marks in Katie's diary identified by the LIWC program (Pennebaker \& Stone, 2004) is intriguing even if we, as yet, can provide no interpretation for this trend.

There is often a conflict between those engaged in, and advocating, the two methodologies. In particular, those who produce qualitative studies often feel that their contributions have greater difficulty getting published and, when published, are often ignored.${ }^{74}$ Antoon Leenaars and his colleagues edited a special issue of the Archives of Suicide Research (2002, volume 6, number 1) in which this conflict was discussed, with proponents of qualitative methodologies (e.g., Gerisch, 2002) and proponents of quantitative methodologies (e.g., de Wilde, 2002) both represented. I argued that the two methodologies could complement each other (Lester, 2002b). For example, Clarke and Lester (2013) showed that the choice of method for suicide is affected greatly by the ease of obtaining the means (such as toxic domestic gas) for that method. On the other hand, case studies, a qualitative method, can indicate why this person chose that method. For example, Grumet (1989), commenting on a male nurse who used an overdose and an electrocution to attempt suicide, noted that the use of electrocution was motivated symbolically by a desire to cure himself of his psychiatric disorder (he had wanted to have electroconvulsive therapy) and as a wish to be punished for being bad (an execution by electrocution).

The analyses in this book, therefore, illustrate the ways in which qualitative and quantitative approaches can complement each other rather than being rival methodologies. Indeed, the analysis of Jim's diary (Chapter 6) used a qualitative analysis of themes, the results from the LIWC computer program, and insights from Jim's sister, providing three different but complementary views of Jim's life.

74 These two approaches parallel to some extent the age-old distinction between idiographic approach (the study of the individual) and the nomothetic approach (the study of samples of individuals). 


\subsection{Poems and Letters}

The study of the poems written by those who died by suicide provided fewer clues into the mind of the writer, probably because poems are crafted carefully by the writer, whereas diaries are rarely changed and edited by their writers. Even though Arthur Inman (Chapter 5) could have edited his diary when his assistants typed the pages, he steadfastly refused to change a word. He wanted his diary to be exactly as he wrote it, even if he changed his mind about what he had written and even if the words insulted or hurt those around him who were typing it. Even Katie's poems (Chapter 15), which not written by an accomplished poet and not intended for publication, gave little insight into the conflicts behind her choice of suicide.

The study of letters (Chapter 16) was also somewhat disappointing. Those of van Gogh revealed little personal information, and those of Clara were written many months before her death by suicide and seemed to be quite superficial in tone. The letters of the young man to a close friend (to whom he was probably attracted) showed some trends consistent with the findings from the studies of the diaries, such as the changes in positive and negative emotions, but the content of the letters gave few clues to his conflicts and his mental pain. Since letters are written for others to read, perhaps the writers are more constrained in what they put down on paper (or write in an e-mail).

Suicide notes are written in the minutes (or hours) prior to death and are less likely to be edited and constrained by the formalities of writing that are present in letters and poems. This is not to argue that we should not study such letters and poems. Those who die by suicide leave so little behind for us to explore the psychodynamics of their choice of suicide that we must use whatever we can find. Occasionally, too, we find a wonderful poem or letter that throws light on the person's choice. For example, Sylvia Plath's poem Daddy (Plath, 1981; see Chapter 1 above) provides clues to her choice of suicide.

The analysis of tweets (Chapter 19) and tape recordings (Chapter 18) also provide uncensored and unedited words from people in the period just before they died by suicide. De Leo's interviews with survivors of suicide attempts (Chapter 21) presented the unedited words of people, and de Leo's sensitive interviewing identified psychological processes that have not been mentioned much in the past (such as the role of anxiety in the moments prior to the suicidal action).

\subsection{Suicide Notes}

Suicide notes have been studied using judges to rate the presence of general themes in many prior studies. For example, Leenaars, et al. (1992b) rated suicide notes for the presence of Karl Menninger's three major themes (anger-in, anger-out, and escape), and Gunn, et al. (2012) rated the presence of the themes from Joiner's (2005) theory of 
suicide (thwarted belonging and perceived burdensomeness). Such studies are often impaired by poor agreement between the raters, and the use of the LIWC program avoids these inconsistencies.

The noteworthy finding of the analyses in Part 2 of this book has been the difference in the results when a large sample of suicide notes is available. In the past, researchers have been constrained by the small samples that they have been able to collect, typically forty or so suicide notes at most. The analysis of the 260 notes from Australia provided much richer results. For example, whereas previous research had identified few, if any, differences in the content of suicide notes written by men and by women, the present analysis in Chapter 13 of the sample of 260 suicide notes identified many differences. The suicide notes written by women referred more to themselves, and contained more positive emotions and references to thought processes and insight (perhaps because they gave more reasons for their choice of suicide).

As noted in Chapter 11, the study of suicide notes was, in my opinion, hindered by the publication by Shneidman and Farberow (1957b) of 33 pairs of genuine and simulated suicide notes. Because of the difficulties of obtaining samples of suicide notes, researchers used these notes for some two dozen studies. I argued that these studies do not throw much light on the suicidal mind but, rather, on lay theories of suicides - what nonsuicidal people think is going on in the mind of the suicidal person. The ideal comparison group should not be simulated suicide notes written by nonsuicidal people, but instead letters and notes written by the suicides a year or more earlier. No researcher has ever attempted to collect such a comparison sample of notes. However, diaries do accomplish this. The last entry prior to the death can be compared with an entry one year earlier. The drawback, of course, is that the sample size is tiny. Part 1 of this book discussed only eight diaries of suicides (if we include Bobby Sands), and I have only recently acquired a ninth diary.

\section{5 "Firsts"}

The present book contained many "firsts."

(1) The study of eight diaries is the first major study of diaries. Lester (2004) provided the first study of one diary, but this book begins the project of collecting a sample of diaries so that we can distinguish general themes from idiosyncratic themes. The inclusion of a diary from a hunger-striker (Bobby Sands in Chapter 10), which showed no resemblance to any of the other diaries, indicated that the diaries of those who die by suicide may have unique properties.

(2) The study of Twitter postings by a young girl written in the hours before her death by suicide (see Chapter 19) illustrated the possibilities that modern media outlets provide for studies of what is transpiring in the mind of the suicide prior to the act. The study of these types of material may be affected by the fact that the individuals know that their Tweets, YouTube video or Facebook posting will be viewed by 
others and, therefore, will be affected by the image that the potential suicides wish to convey but this is true for suicide notes also. Yang and Lester (2011) argued that we should not always believe the truthfulness of the content in suicide notes since writers of such notes are often trying to shape their image so that they are remembered in a particular way by their significant others.

(3) The study of the two tape recordings made the young man in the hours before he died by suicide (Chapter 18) provide the first ever insight into the mind of potential suicides immediately prior to their deaths. As I noted in Chapter 18 (and throughout the book), two research teams had reported that the mood of the suicide seemed to improve in the hours prior to their death, and this was clearly documented in the two tapes left by the young man. The second recording was calmer and more otheroriented than the first tape.

(4) The discussion of Diego de Leo's (2010) book (Chapter 21) reporting his interviews with those who made serious attempts to die by suicide, but who failed, shows how little insight that individuals have into their own minds. The more formal analysis of the written and spoken words of suicidal individuals provides much more information. However, as I noted in Chapter 21, where I reviewed the accounts of these attempted suicides, the role of anxiety in the minutes prior to their suicidal act came through clearly in their accounts, and this had not been identified by the more formal research conducted on attempted suicides in the hours and days after their actions using standardized psychological tests.

\subsection{Understanding the Mind of the Suicidal Individual}

Although those who are suicidal or about to attempt or complete suicide can tell us what the precipitating event is, they are often unaware of the psychodynamics behind their act. In scholarly terms, they can tell us about the stress but not the diathesis. Even the most popular theory of suicide in recent years, that proposed by Joiner (2005), can tell us only about the triggering events (thwarted belongingness and perceived burdensomeness), but nothing about why some people react to a thwarted relationship by killing themselves while most do not. These theories of suicide also tell us very little about what is going on in the mind of the suicidal person. We should note in passing that, even when suicidal individuals do have some insight, as in the case of Sylvia Plath, this does not prevent their suicide.

The words of the suicides, written or spoken, clearly attest to the immense psychological distress in their minds. There is a world of difference between a word like Shneidman's (1996) psychache and the expression of this pain in Katie's diary (Chapter 3) or Stephen's spoken words (Chapter 18). For some of the people discussed in this book, this pain seems to lessen in the hours (Stephen) or days and weeks (Katie) before the suicide but, prior to that, the pain is raw. 
It does appear that we have underestimated the role of anxiety in the moments prior to a suicide. De Leo's interviews with survivors of attempted suicide documented this anxiety, but so does the diary of Arthur Inman (Chapter 5). Although the published diary omits the entries from the final few months, I have perused the handwritten entries for the final month and can attest to the presence of acute anxiety in Inman in the days before he killed himself.

The existence of schemata in which one thought leads to another and then another wa proposed by Kovacs and Beck (1978), and this was evident in the diaries of Cesare Pavese (Chapter 4) and Robert (Chapter 7). In Pavese's case, failure in love led to thoughts that he was a failure as a writer. In Robert's case, anxiety over his teaching and scholarly work led to thoughts of his Oedipal complex and sexual conflicts. In addition, in Robert's case, the way in which one pejorative word about himself led to a seemingly well-rehearsed sequence of such words was noteworthy.

Cognitive theories of suicide have often noted the tunnel vision (cognitive constriction) and rigidity in the thinking of suicidal people. This was evident, for example, in the diary of Inman who saw no alternative to his living situation. Either he had to stay where he was living, or he had to kill himself. However, it is interesting to see how Jim (Chapter 6) also constructed the situation so that he had no other choice. He killed himself when he ran out of money. His electricity was about to be cut off, his house would be seized, and he had no money left for food or alcohol - not one cent. Jim constructed the cost-benefit analysis of the decision to die by suicide so that the benefit outweighed the cost.

It is important to note that the suicides discussed in this book are more dissimilar from one another than similar. Although it is possible to draw some parallels, they are very different. Katie has romantic relationship problems, but Pavese has no romantic relationships (only fantasies). Inman cannot tolerate situational change (moving house), while Robert cannot tolerate a negative academic judgment (a failure to obtain tenure). Inman is anxious, but Jim is resigned.

Even when there are similarities, there are slight differences. Pavese's diary showed an increase in positive emotions over the last few months of the diary but, in the letters from a young man to his friend (Chapter 16), there was a decrease in negative emotions. The overall mood in both improved, but for different reasons. There are, therefore, perhaps no generalizations we can draw about every suicide. Not surprisingly, each is unique.

Finally, it is interesting to note that theories of suicide have little to say about the suicides presented and discussed in this book. Instead, the classic theories of personality (such as psychoanalytic theory) and the classic systems of psychotherapy (such as cognitive therapy) provide a better framework for analyzing and understanding these suicides. 


\subsection{Final Thoughts}

The present book has shown the importance of "listening" to the words that those about to die by suicide say and write. Our formal psychological tests provide useful information and enable us to test our theories of suicide, but they fail to capture the pain and the thought processes of the suicidal person. Biographies of suicides and clinical reports of cases are, of course, interesting, but they are far removed from the experiences of the protagonists. Only their own words can give us clues as to what is transpiring in the "I" of the storm. 